\title{
A AQUISIÇÃO DE VOCABULÁRIO POR INTERMÉDIO DA LEITURA
}

\author{
Maria Amélia Quélhas Moreira
}

Mestranda em Língua Inglesa pela UFPR

\begin{abstract}
RESUMO: O presente artigo descreve o resultado de pesquisas na área de aquisição de vocabulário em segunda língua por intermédio da leitura e enfatiza a importância do contexto de leitura como elemento facilitador da aprendizagem incidental de vocabulário. Das quatro habilidades que precisam ser dominadas para que um indivíduo se torne proficiente em uma segunda língua - falar, ouvir, ler e escrever - a leitura é considerada por muitos a mais importante, especialmente por ser a fonte de input mais facilmente acessível. As pesquisas têm demonstrado que existe uma relação causal direta entre vocabulário e leitura: uma competência lexical bem desenvolvida é um fator determinante para a compreensão da leitura e, por outro lado, a leitura é o veículo primordial para o desenvolvimento do vocabulário. A expansão do vocabulário por intermédio da leitura é o escopo desse trabalho, que relacionará a aprendizagem incidental de vocabulário a partir da leitura a outros elementos que podem potencializar o uso do contexto: a instrução direta de vocabulário, o ensino de um vocabulário básico e o uso do dicionário.
\end{abstract}

\section{Introdução}

Nas duas últimas décadas, a aquisição de vocabulário vem recebendo, grande atenção da pedagogia e pesquisa em segunda língua (L2 doravante), refletindo, dessa forma, a importância que os próprios aprendizes atribuem a esse tema. Na maioria das vezes a palavra "vocabulário" é diretamente relacionada à listas de palavras, na suposição de que aprender vocabulário signifique, somente, memorizar tais listas. Conforme Meara (1980) ressaltou, porém, tais suposições

(...) "ignoram completamente modelos complexos de relações de significado que caracterizam um léxico apropriado e plenamente formado, em oposição à mera lista de palavras." (1c)

A afirmação acima ilustra a importância que o léxico tem adquirido como elemento crucial para a aquisição de uma segunda língua. Segundo Paribakht e Wesche (1997), existem consideráveis evidências que demonstram ser a leitura a principal responsável pela expansão do vocabulário em língua nativa. A pesquisa em segunda língua sobre esse assunto é esparsa, mas há suficientes indicativos de que programas de leitura extensiva (leitura menos rigorosamente supervisionada e que seja do interesse dos alunos) são geralmente mais efetivos do que a instrução sistemática de vocabulário, usando exercícios descontextualizados.

Nick Ellis (1995) identificou os processos através dos quais adquirimos vocabulário enquanto lemos. Segundo Ellis, as teorias de aquisição de vocabulário oferecem uma gama de hipóteses que vão da aprendizagem implícita àquelas que argumentam que aos alunos devam ser ensinados, explicitamente, grandes quantidades de vocabulário.

Há várias possibilidades de como a aquisição de vocabulário ocorre:

1. Uma hipótese forte de aprendizagem implícita de vocabulário estabelece que o significado de uma palavra nova é adquirido de forma totalmente inconsciente como resultado de abstração a partir de repetidas exposições em vários contextos. 
2. Talvez a aquisição não seja possível sem que o leitor "note" que tal palavra é nova (Schmidt, 1990), o que caracteriza uma hipótese fraca de aprendizagem implícita de vocabulário.

3. As pessoas são ativos processadores de informações. A hipótese de aprendizagem implícita considera que adquirimos a maioria das palavras que conhecemos subconscientemente, elas não nos foram ensinadas. Mas isso não significa que nós não tenhamos ensinados a nós mesmos. Desse modo, uma hipótese fraca de aprendizagem explícita de vocabulário assegura que o leitor que "nota" uma palavra nova facilita a sua aquisição de vocabulário ao prestar atenção a essa palavra e ao usar diferentes estratégias para tentar inferir o seu significado pelo contexto.

4. Uma hipótese forte de aprendizagem explícita de vocabulário assegura que a aplicação de uma grande variedade de estratégias metacognitivas facilita grandemente a aquisição de vocabulário novo:

- notando-se que a palavra não nos é familiar.

- fazendo tentativas para inferir seu significado pelo contexto ou chegando a ele consultando outras pessoas ou dicionários.

- fazendo tentativas para consolidar esse novo conhecimento através da repetição e de estratégias de aprendizagem associativas tais como: técnicas de mediação semântica ou de imagens.

O objetivo deste trabalho é discutir a relação existente entre vocabulário e leitura, enfatizando alguns dos aspectos que permitem uma maior aquisição de vocabulário por intermédio da leitura, tais como:

- a aprendizagem incidental de vocabulário a partir da leitura;

- a aprendizagem a partir da leitura combinada à uma instrução explícita e sistemática de vocabulário; 
- o ensino de um vocabulário básico (core vocabulary) (Carter, 1987) de cerca de 2.000 palavras de alta freqüência que permita o uso da leitura como forma de expandir o vocabulário;

- o uso de dicionários como recurso adicional que possibilite uma maior retenção do vocabulário aprendido.

\section{A Aprendizagem Incidental de Vocabulário por Intermédio da Leitura}

A maioria das palavras que conhecemos não nos foi ensinada e nem as procuramos no dicionário; a maioria do vocabulário é aprendida a partir do contexto (Sternberg, 1987). A leitura é considerada um meio ideal para a aquisição de vocabulário porque as palavras de baixa ou moderada freqüência aparecem muito mais freqüentemente em textos de leitura comuns do que $\mathrm{n}$ a conversação habitual. O leitor tem a oportunidade de estudar o contexto, estabelecer hipóteses e confirmá-las, e inferir significados, com calma. A palavra está congelada no tempo, no papel, enquanto que a fala flui rapidamente. Segundo Stanovich e Cunningham (1992), as pessoas que lêem mais conhecem mais vocabulário.

A aquisição de vocabulário é vista, por diferentes autores, de diferentes maneiras. O uso da leitura como forma de aquisição de vocabulário configura uma abordagem indireta de ensino de vocabulário em que a atenção do aluno está voltada para o assunto de que o texto trata e, não, especificamente, para o vocabulário a ser adquirido. Porém, conforme Scaramucci (1995) ressalta, "há uma distinção entre a construção do significado através de pistas contextuais e o real aprendizado e retenção desses significados." Os diversos estudos na área de aquisição de vocabulário mostram que usar as pistas disponíveis no contexto para inferir o significado das palavras desconhecidas, não implica ter tais palavras, automaticamente, incorporadas ao nosso léxico mental. Alguns estudos, como o de Parry (1988) e Nagy e Herman (1987), mostram que nem sempre as palavras inferidas a partir do contexto são aprendidas. Por outro lado, há várias evidências de que o vocabulário pode, de fato, ser aprendido em contexto. Nagy e Anderson (1984), em defesa da aprendizagem de vocabulário através do contexto, enfatizam que

\section{(...) "nem mesmo o ensino de vocabulário mais direto e implacavelmente sistemático pode explicar uma proporção significativa de todas as palavras que as crianças realmente aprendem, nem cobrem mais do que uma modesta proporção das palavras que elas encontrarão nos materiais de leitura escolares." (2c)}

Krashen (1989) é o principal proponente da leitura extensiva como meio de aquisição de vocabulário. Para Krashen (1989) a competência em vocabulário é alcançada mais eficientemente através de input compreensível (confira Krashen, 1985) e é a leitura a melhor 
forma de input compreensível para o desenvolvimento do vocabulário, tanto em relação à $L 1$ quanto à L2. Desse modo, o processo de aquisição é incidental, já que a aquisição de vocabulário ocorre sem aprendizagem, ou seja, sem nenhum tipo de instrução formal. De acordo com a teoria de Krashen, o objetivo é criar condições que facilitem a "aquisição" inconsciente e minimizem o tempo gasto na aprendizagem consciente das propriedades formais da língua. Desse modo, o processo de "aquisição" é idêntico à "aprendizagem incidental de vocabulário", hipótese formulada por Nagy e Herman (1985), a qual se baseia em pesquisa sobre como as crianças aprendem vocabulário em suas línguas maternas. Eles consideram que progressos importantes na aquisição de vocabulário só podem ser conseguidos aumentando-se a aprendizagem incidental de vocabulário através de um contexto de leitura. Nagy e Herman afirmam que a maioria das palavras são aprendidas, gradualmente, através de repetidas exposições em vários contextos lingüísticos. De fato, a repetição leva à prática e Nick Ellis (1995) afirma que "é a prática que faz a perfeição nos módulos de reconhecimento e produção" (3c). Tal afirmação é confirmada por Hulstijn (1992) ao concluir, em vários estudos com aprendizes adultos de L2, que a retenção do significado das palavras, em uma única tarefa de aprendizagem incidental é muito baixa. Nagy, Herman e Anderson (1985) anteciparam as conclusões de Hulstijn, mas reafirmaram a importância da aprendizagem incidental ao salientarem, com base em experiência com diferentes livros textos, que mesmo quando uma palavra desconhecida é vista impressa em uma página uma única vez, "um aumento do conhecimento lexical, pequeno mas estatisticamente confiável" (4c), ocorre.

Nagy, Anderson e Herman (1987) observam que, embora a probabilidade de aprender uma palavra através do contexto seja muito pequena para ter algum valor prático, não podemos deixar de considerar a quantidade de leitura a que os alunos são expostos, a fim de poder avaliar, corretamente, a contribuição da aprendizagem pelo contexto, através da leitura, em relação à expansão do conhecimento lexical, a longo prazo: mesmo uma pequena probabilidade de aprendizagem de uma palavra através do contexto pode resultar em uma grande expansão do vocabulário, se houver quantidade suficiente de leitura ampla e abrangente. Para Nagy et al. (op. cit.), a aprendizagem através do contexto da leitura ocorre em pequenos incrementos de modo que qualquer encontro com uma palavra resulta, geralmente, em um pequeno aumento do conhecimento das diferentes acepções daquela palavra.

A clássica experiência de Saragi, Nation e Meister (1978), com a leitura do livro de Anthony Burgess, "A Clockwork Orange", nos revela que a simples repetição de palavras desconhecidas em um texto (seus estudos sugerem que o número mínimo de repetições deva ser de cerca de 10 vezes) é suficiente para que a aquisição de vocabulário aconteça. A novela de Burgess contém várias palavras novas de origem russa chamadas "nadsat". São 241 palavras "nadsat" que são repetidas uma média de 15 vezes na novela. Os leitores foram solicitados a ler o texto simplesmente, sem nenhuma referência ao significado das palavras novas. Depois de alguns dias, um teste surpresa foi feito. Houve considerável aquisição de vocabulário, já que os leitores retiveram 45 das 90 palavras "nadsat" testadas.

O ponto mais importante a favor da aprendizagem incidental através da leitura, na opinião de vários autores, foi apontado por Nagy (1988) ao constatar que a leitura resulta em um conhecimento profundo do vocabulário; em textos autênticos, as palavras são encontradas em uma grande variedade de contextos, o que permite ao aluno assimilar suas propriedades semânticas e sintáticas de uma maneira muito mais profunda. Mais importante ainda é considerar que o encontro com essas palavras novas em contexto (considerando-se que o restante do texto seja compreensível) permite ao leitor estabelecer ligações entre a informação 
nova e as informações já existentes, o que, certamente, o ajuda a implantar esse novo conhecimento em sua memória de longo prazo.

\section{A Aprendizagem por Intermédio da Leitura Combinada a uma Instrução Sistemática de Vocabulário}

Paribakht e Wesche (1997), embora reconhecendo que a leitura extensiva conduz à aquisição de vocabulário, apresentam estudos que demonstram que a aprendizagem através da leitura combinada a uma instrução sistemática de vocabulário é uma abordagem mais bem sucedida do que a simples aprendizagem através do contexto. Elas realizaram uma pesquisa a fim de avaliar até que ponto a leitura, somada a atividades de instrução de vocabulário, poderia resultar e m ganhos para o leitor em termos de uma maior retenção de vocabulário. Os leitores foram submetidos a dois tratamentos diferenciados:

1. Reading Plus (RP): Os alunos liam os textos selecionados e depois respondiam a perguntas de compreensão. Em seguida, faziam exercícios de vocabulário sobre as palavras alvo, previamente selecionadas no texto. Os exercícios de vocabulário foram hierarquizados levando-se em consideração a "profundidade de processamento" (depth of processing) (Craik e Lockhart, 1972) necessária para a sua realização. Desse modo, os exercícios foram agrupados em cinco categorias distintas, representando uma hierarquia da atividade de processamento mental:

- Atenção seletiva: Com esses exercícios o objetivo é assegurar que o aluno "note" a palavra alvo,

- Reconhecimento: Os alunos são solicitados, somente, a reconhecer a palavra alvo e seu significado,

- Manipulação: Exercícios de manipulação envolvem a reorganização de determinados elementos para formar novas palavras ou frases (usando, por exemplo, afixos),

- Interpretação: Envolve a análise dos significados das palavras com respeito a outras palavras em contextos diferentes (colocações, sinônimos e antônimos) e 
- Produção: Esse tipo de exercício requer que o aluno produza as palavras alvo em contextos apropriados.

2- Reading Only (RO): Os alunos liam os textos e respondiam a perguntas de compreensão. Em seguida, ao invés de exercícios de vocabulário, liam textos suplementares onde as palavras alvo eram novamente usadas. Mais uma vez, respondiam a perguntas de compreensão sobre o texto lido.

Usando-se a escala VKS (Vocabulary knowledge Scale), os ganhos em termos de aumento do conhecimento lexical eram, então, medidos levando-se em conta um tratamento e o outro. A escala VKS mede o conhecimento lexical, variando do total desconhecimento da palavra ao reconhecimento da palavra e alguma idéia do seu significado até a habilidade de usar a palavra numa sentença com precisão gramatical e semântica.

Ao analisarem os resultados, concluiu-se que os dois tratamentos produziram ganhos significativos, mas o tratamento RP resultou em ganhos maiores do que o tratamento RO, tanto quantitativos quanto qualitativos. Muitos leitores no tratamento RP passaram do nível de reconhecimento das palavras alvo e alcançaram uma maior profundidade no conhecimento destas palavras. $O$ resultado desse estudo indicou que os leitores, no tratamento $\mathrm{RO}$, adquiriram algum vocabulário novo, mas o conhecimento de muitas dessas palavras tenderam a permanecer em nível de reconhecimento. De uma maneira geral, os alunos tendem a ignorar o significado de palavras desconhecidas, a menos que elas sejam essenciais para que eles possam alcançar um nível desejável de compreensão do texto. Essa estratégia, embora justificável em relação à prática geral da leitura, não é ideal para o enriquecimento do vocabulário dentro de um período de instrução limitado. Por outro lado, no tratamento RP os leitores aprenderam um número maior de palavras e seus conhecimentos atingiram níveis mais altos de processamento.

Em conclusão, Paribakht e Wesche (op. cit.) afirmam que, embora a leitura que vise à compreensão do texto possa produzir resultados incidentais significativos na aquisição de vocabulário, tal leitura, suplementada com exercícios específicos de vocabulário, produz ganhos maiores em relação às palavras alvo. A razão para o sucesso da leitura seguida de exercícios de vocabulário é que esses exercícios garantem que a atenção do aluno seja focalizada em itens específicos de vocabulário o que leva os alunos a analisar e compreender os significados e funções das palavras alvo através de tarefas variadas.

\section{O Ensino de um "Core Vocabulary"}

Apesar dos argumentos acima mencionados, e de acordo com Laufer (1997), não se pode pressupor que inferência em segunda língua seja realmente possível com a maioria das palavras desconhecidas e que uma inferência bem sucedida dependa, exclusivamente, das estratégias usadas pelo aluno. Haynes e Baker (1993) chegaram à conclusão de que a mais importante dificuldade para leitores de uma segunda língua não é a falta de conhecimento de 
estratégias de leitura (ou das estruturas gramaticais básicas), é, sim, um vocabulário insuficiente na língua alvo. A porta de entrada para a compreensão da leitura é, definitivamente, lexical.

Laufer (op. cit.) afirma que quando um leitor em segunda língua, com um vocabulário insuficiente, tenta interpretar um texto autêntico, ele, normalmente, será incapaz de aplicar as estratégias de leitura que ele usa ao ler um texto em sua língua nativa. Para que possa fazer uma correta transferência dessas estratégias é necessário que o leitor possua o que Laufer chama de vocabulário limiar (threshold vocabulary), que também é chamado de sight vocabulary, palavras cujas formas e significados comuns são reconhecidos automaticamente, independentemente do contexto. O reconhecimento automático de um vocabulário básico liberta os recursos cognitivos do leitor para que possa tentar inferir o significado de palavras pouco ou não conhecidas e para interpretar a mensagem global do texto. Se o leitor despende um esforço cognitivo muito grande para decifrar informações em nível da frase (ao tentar adivinhar o significado de palavras desconhecidas, por exemplo), ele terá dificuldades para identificar as relações existentes entre os parágrafos. Desse modo, quando ele atingir o final do segundo parágrafo, já terá esquecido as informações contidas no primeiro e ficará impossibilitado de fazer as devidas conexões entre os dois. Perfetti e Lesgold (1977, 1979 apud Coady, 1993) argumentam que, quando o esforço do leitor ao reconhecer uma palavra é lento e trabalhoso, sua memória de curto prazo fica tão sobrecarregada que o leitor não consegue se beneficiar plenamente do contexto. É necessário, portanto, que o leitor possua um vocabulário de reconhecimento automático que seja suficiente para que ele possa se beneficiar do contexto e fazer as inferências corretas.

Segundo Nation e Newton (1997), as 2.000 palavras de alta freqüência em Inglês (e, naturalmente, em quaisquer outras línguas) devem receber atenção imediata porque sem elas é impossível usarmos a língua em qualquer situação normal. Ao selecionarmos o vocabulário básico para aprendizagem devemos levar em consideração, não apenas a freqüência e 0 alcance das palavras, mas também suas habilidades de combinar-se, definir ou substituir outras palavras. Esses fatores e outros se combinaram na noção de core vocabulary.

Conforme vem sendo demonstrado neste trabalho, a aprendizagem de vocabulário pode ocorrer através da exposição a textos diversos. Pesquisas como a de William Grabe (Grabe e Stoller, !997), em experiência realizada quando passou 5 meses no Brasil em 1990, confirmam que várias exposições de diferentes intensidades conduzem, gradualmente, a uma expansão do vocabulário de reconhecimento. Entretanto, devemos prestar atenção especial ao problema que alunos iniciantes enfrentam e que Coady (1997) denominou "o paradoxo do iniciante":

"Como é possível que alunos iniciantes possam aprender palavras suficientes para serem capazes de aprender vocabulário através da leitura extensiva se eles não conhecem palavras suficientes para ler bem?" (5c)

A resposta à pergunta de Coady está certamente no ensino de um core vocabulary. Para Laufer (op. cit.), o conhecimento de vocabulário necessário à compreensão mínima de um texto de leitura seria de 3.000 famílias de palavras, que contêm cerca de 5.000 itens lexicais. De acordo com Nation e Newton (1997), já que as palavras de alta freqüência são relativamente poucas e são essenciais para o uso efetivo da língua, cada uma delas merece atenção por parte do professor. As palavras de baixa freqüência, por outro lado, são muitas, podem, constantemente, ser inferidas pelo contexto (se as palavras de alta freqüência forem conhecidas) e não ocorrem 
freqüentemente, por isso não merecem atenção especial do professor. Contudo, estratégias para que os alunos possam lidar com essas palavras e aprendê-las merecem consideração especial por parte do professor. Essas estratégias incluem a inferência pelo contexto, o reconhecimento de afixos (sufixos e prefixos), que possibilita a dedução do significado da palavra nova, e o uso de estratégias de aprendizagem mnemônicas e de repetição. A principal preocupação do professor deve ser o desenvolvimento efetivo dessas estratégias.

Ainda segundo Nation e Newton, o ensino de vocabulário pode seguir uma abordagem direta ou indireta, e essas abordagens não precisam ser alternativas mas, sim, complementares. Numa abordagem direta, atenção explícita é dada ao ensino de vocabulário com lições, exercícios e testes regulares de vocabulário. Numa abordagem indireta, a preocupação do professor com o ensino de vocabulário não precisa ser tão óbvia. O professor pode incorporar o ensino de vocabulário à atividades comunicativas e incentivar a leitura simplificada (leitura de graded readers). O ensino de vocabulário pode ser um objetivo incidental ou mesmo um dos objetivos principais de uma atividade de comunicação, em que a atenção se concentre no desempenho interativo em uma atividade comunicativa e não na linguagem propriamente usada. Nation e Newton citam exemplos do uso de exercícios interativos nos quais os alunos trocam informações que precisam ser negociadas, tanto quando eles compartilham as mesmas informações (shared information) e precisam usá-las para resolver um problema ou tomar decisões, quanto quando um possui uma informação desejada pelo outro (split information). Hall (1992) sugere que a necessidade de um output oral e o uso gerativo de novos itens lexicais (seu uso em novos contextos e em novas estruturas) são fatores preponderantes que levam à aquisição desse novo vocabulário.

No exercício sugerido no apêndice do artigo de Nation e Newton os alunos são solicitados a transferir informações de uma tabela para outra e, depois, avaliar criticamente e categorizar as informações de acordo com alguns critérios. Ao incorporar dois tipos de desempenho numa mesma atividade, os alunos são forçados a obter precisão e fornecer informações corretas e também a participar de discussões que os levarão a tomar as decisões mais apropriadas. $\mathrm{Na}$ atividade acima mencionada, podemos perceber que o aluno pode desenvolver indiretamente seu vocabulário produtivo e também o de reconhecimento, como resultado da exposição a novas palavras num contexto comunicativo significativo. Pode, igualmente, desenvolver seu vocabulário diretamente, como resultado de um trabalho comunicativo com um vocabulário alvo.

É muito importante adquirir vocabulário novo, mas é também essencial ser capaz de acessá-lo rapidamente no nosso léxico mental, quando precisamos usá-lo. Há duas maneiras pelas quais o vocabulário pode ser ensinado, de forma a ser acessado prontamente: através de atividades de fluência (fluency activities) ou atividades de enriquecimento (richness activities). As atividades de fluência são aquelas que envolvem o processamento de uma grande quantidade de linguagem, usam material que não contenha muita linguagem desconhecida, envolvem preparação e planejamento. São atividades que atingem um alto grau de automaticidade. As atividades de enriquecimento têm como objetivo aumentar o número de associações que acompanham uma palavra. Elas podem ser de dois tipos: aquelas que estabelecem relações sintagmáticas e aquelas que estabelecem relações paradigmáticas. Relações sintagmáticas são aquelas que associam uma palavra com outras palavras que podem precedê-la ou segui-la. Por exemplo: em relação à palavra fuel temos as associações alternative fuels, fuels inflation ou a frase add fuel to the flames. Relações paradigmáticas são aquelas que associam uma palavra com outras cujos significados estejam a ela relacionados. Por exemplo: não é apenas relevante que table signifique um objeto físico no mundo real, mas que a palavra table possa ser 
sistematicamente relacionada às palavras chair, sofa, desk e furniture no sistema da língua.

Para finalizar é bom lembrar que as redes associativas que existem na língua nativa são construídas, para o falante, gradativamente, através do uso normal da língua. Pesquisas revelaram que a aprendizagem conjunta de vocábulos que sejam "quase" sinônimos ou antônimos é muito mais difícil do que a aprendizagem de vocábulos sem nenhuma relação entre si. Desse modo, ensinar hote cold ao mesmo tempo torna a retenção dos significados mais difícil, porque muitos alunos irão misturar as formas e os significados das palavras. Da mesma forma, aprender vocábulos como shrewd, sly, cunning, crafty, ao mesmo tempo, resulta em mais confusão do que clareza e dificulta a aprendizagem. A hora certa para se fazer tais associações é quando todos os vocábulos do grupo, menos um deles, são bem familiares e precisa-se, apenas, esclarecer as distinções entre eles.

\section{O Uso de Dicionários}

Segundo Summers (1988), o uso de dicionários é um recurso válido para aprendizes de uma língua estrangeira, tanto como um meio de auxiliar a compreensão quanto a produção. Além do uso de outras estratégias de aprendizagem, (tais como: fazer inferências sobre palavras novas encontradas nos textos de leitura, pedir explicações ao professor ou pedir ajuda aos colegas), os alunos podem e devem ser encorajados a se beneficiar das substanciais informações contidas num dicionário.

Contrário às afirmações acima, o princípio pedagógico que prevalece é que palavras novas devam ser decodificadas, apenas, a partir das pistas fornecidas pelo contexto. Na realidade, muito freqüentemente, as palavras desconhecidas dentro de um texto não podem ser deduzidas através das pistas contextuais. Tentar inferir o significado de palavras desconhecidas num texto é uma estratégia importante para a compreensão da linguagem usada no texto, mas o uso do dicionário também é um recurso valioso.

Segundo Grabe (1997), inferência em excesso leva a uma grande frustração porque há momentos em que é importante saber se determinada palavra foi entendida com precisão. Grabe, em sua experiência no Brasil, afirma que a consulta a dicionários é um elemento importante na aquisição de vocabulário. Ele verificou que um nível alto de frustração ocorre quando o leitor conta basicamente com a inferência do significado de palavras desconhecidas a partir do contexto. Os leitores têm necessidade de saber que os significados das palavras estão corretos para que eles possam continuar lendo com um certo grau de confiança. Mas é sempre preferível que os alunos cheguem ao significado das palavras de um texto a partir das pistas que ele fornece, pois existe sempre a suspeita de que o uso do dicionário interrompe o fluxo de concentração quando o aluno está lendo um texto. Entretanto, quando os significados de algumas palavras não podem ser inferidos a partir das pistas do contexto, é importante encorajar os alunos a fazer uso do dicionário. O dicionário também oferece ajuda em relação às colocações, às relações sintagmáticas das palavras e às suas possibilidades gramaticais. Além disso, a atividade mental envolvida na elaboração intelectual da definição dada pelo dicionário, ajuda a implantar a palavra e seu conceito na memória do aluno, especialmente quando a definição apóia-se em exemplos explicativos. Summers (1988) argumenta que, embora o texto propriamente dito possa fornecer pistas sobre o significado de palavras desconhecidas, quando alunos e professores precisam de informações para fins de produção ( para a redação de um ensaio, por exemplo), o dicionário pode ser a única ajuda disponível. É bastante improvável que 
os alunos possam inferir colocações de palavras a partir de um ou dois contextos dos quais eles possam se lembrar ou, ainda, deduzir regularidades gramaticais. Nesse caso, o mais provável é que eles revertam à sua língua nativa e acabem cometendo erros de interferência.

Luppescu e Day (1995) realizaram uma pesquisa com a finalidade de avaliar o papel dos dicionários na aprendizagem de vocabulário durante a leitura. Nela eles contrapõem aquisição de vocabulário apreendido pelo contexto com aquele apreendido através de consultas a dicionários. Os participantes do estudo foram universitários japoneses com 6 anos de estudo de Inglês nas escolas secundárias, com bons conhecimentos gramaticais, mas pouca habilidade comunicativa. Os participantes foram colocados, aleatoriamente, em dois grupos: o grupo de controle e o grupo de tratamento. Os alunos do grupo de tratamento leram o texto e tiveram permissão para usar dicionários bilíngües e os do grupo de controle leram a mesma história, mas não tinham permissão para usar dicionários. A comparação da quantidade de vocabulário assimilada por cada grupo baseou-se nos resultados de um teste de vocabulário realizado pelos dois grupos.

A hipótese era de que não haveria diferença significativa na quantidade de vocabulário apreendido pelos participantes que fariam uso de dicionários e aqueles que não os utilizariam. Tal hipótese se baseava em duas considerações. Primeiro nas afirmações de Krashen (1982) que salienta que vocabulário que é adquirido naturalmente é mais persistente e mais fácil de lembrar do que aquele aprendido explicitamente, através da memorização ou do uso de dicionários. Em segundo lugar, muitos aprendizes de uma língua estrangeira já tiveram a experiência de verificar que muitas palavras, procuradas no dicionário, são rapidamente esquecidas. Uma segunda hipótese seria de que os alunos que fariam uso do dicionário levariam um tempo muito maior para ler o texto do que os outros que usariam, apenas, o contexto para inferir significados.

As seguintes conclusões a que eles chegaram são, em muitos casos, surpreendentes:

- O grupo que usou dicionário reteve um número maior de palavras do que o grupo que se beneficiou, apenas, das pistas fornecidas pelo contexto. Tal afirmação foi confirmada, mesmo quando um outro teste (de avaliação do desempenho do dois grupos) foi dado três semanas depois que os alunos haviam lido o texto.

- Por outro lado, em alguns casos, o uso do dicionário inibiu a aprendizagem de vocabulário porque o grande número de entradas no dicionário, provavelmente, confundiu o aluno, que não foi capaz de chegar à acepção correta de uma determinada palavra. Isso os levou à recomendação de que se dê instrução explícita e prática do uso de dicionários em sala de aula.

- O grupo que utilizou dicionários levou quase duas vezes mais tempo para ler o texto, o que fornece evidência empírica de que o uso de dicionários diminui a velocidade da leitura.

Pode-se argumentar, porém, que o maior tempo consumido pelo grupo que usou 
dicionários é, justamente, o que justifica os melhores resultados por eles obtidos.

- Uma descoberta inesperada, mas interessante, é que os alunos que usaram dicionários responderam I don't know (uma das alternativas de respostas no teste) com uma freqüência muito menor do que os outros. Esse fato confirma a idéia de que o texto, propriamente dito, não era suficientemente transparente para que os alunos pudessem inferir o significado de todas as palavras e o uso do dicionário, para esclarecer o significado ambíguo de algumas palavras, poderia ter sido de grande utilidade.

Para finalizar, é importante esclarecer que a pesquisa acima descrita foi realizada com dicionários bilíngües. O objetivo da pesquisa foi avaliar o papel de dicionários na aprendizagem de vocabulário, não levando-se em consideração se bilíngües ou monolíngües. Contudo, alguns lingüistas como Baxter(1980, apud Luppescu e Day, op. cit.) são de opinião que o uso continuado de dicionários bilíngües é prejudicial ao desenvolvimento da proficiência na segunda língua. Ele enfatiza que o uso de um "English Learner's Dictionary" (monolíngüe) é importante porque

(...) "não somente demonstra que definição é uma alternativa ao uso de itens lexicais, como também fornece os meios para, de fato, usarmos definição". (6c)

\section{Conclusão}

A leitura é, sem dúvida, a fonte de input mais facilmente disponível para a maioria das pessoas, não somente para aprendermos palavras novas numa língua estrangeira, como também como forma de enriquecermos nosso vocabulário em nossa língua nativa. Contudo, não é possível compreendermos um texto, seja em nossa língua nativa ou numa língua estrangeira, sem compreendermos o vocabulário desse texto. Segundo Laufer (1997), isso não significa dizer que compreensão de texto e compreensão de vocabulário sejam a mesma coisa ou que a qualidade da leitura seja determinada, apenas, pelo vocabulário. A compreensão de um texto é afetada pelo conhecimento prévio (background knowledge) do leitor, relativo ao assunto de que o texto trata e pela aplicação de estratégias gerais de leitura, tais como: prever o conteúdo do texto, inferir o significado de palavras desconhecidas pelo contexto, reconhecer o tipo e a estrutura do texto e compreender a idéia central de um parágrafo.

Segundo Haynes e Baker (1993), porém, quaisquer que sejam os efeitos do uso de estratégias de leitura, elas demonstraram ser totalmente ineficientes se o aluno não possuir um vocabulário básico. Esse vocabulário pode ser mais prontamente adquirido, diretamente, através de exercícios para a fixação do vocabulário, introduzido através de leitura simplificada ou, indiretamente, através de atividades comunicativas, ou, ainda, usando-se o dicionário como forma de acessar vocabulário que não possa ser, facilmente, deduzido pelo contexto. Entretanto, a partir da aquisição de um vocabulário básico, é a leitura, com conseqüente aquisição de vocabulário incidental, que se constituirá em um meio poderoso para o enriquecimento do nosso 
léxico.

Este trabalho demonstrou que vocabulário e leitura, conforme sugerem Grabe e Stoller (1997), são reciprocamente causais e que não há dúvida,portanto, sobre a importância do conhecimento de vocabulário para se efetuar uma leitura suficiente e, por outro lado, do valor da leitura como um meio de adquirir vocabulário.

\section{Citações}

$1 \mathrm{c}-$ "... completely ignore the complex patterns of meaning relationships that characterise a proper, fully formed lexicon, as opposed to a mere word list."

2c- "... even the most ruthlessly systematic direct vocabulary instruction could neither account for a significant proportion of all the words children actually learn, nor cover more than a modest proportion of the words they will encounter in school reading materials."

3c- - "... it is practice that makes perfect in the input and output modules."

4c- - "... a small but statistically reliable increase in word knowledge."

5c- "How can they (beginners) learn enough words to learn vocabulary through extensive reading when they do not know enough words to read well?"

$6 c-" .$. not only demontrates that definition is an alternative to the use of lexical items, but it also provides the means to actually employ definition."

Referências Bibliográficas:

CARTER, Ronald. Vocabulary: Applied Linguistics Perspectives. London: Allen e Unwin, 1987.

COADY, James. Research on ESL/EFL vocabulary acquisition: Putting it in context. In Huckin, T., Haynes M. \& Coady,J. (Eds.) Second Language Reading and Vocabulary Learning. Norwood, NJ: Ablex, 1993.

COADY, James. L2 vocabulary acquisition through extensive reading. In Coady, J. \& Huckin, T. (Eds.) Second Language Vocabulary Acquisition. Cambridge: Cambridge University Press, 1997.

CRAIK, F. I. \& LOCKHART, R. S. Levels of processing: A framework for memory research. In Journal of Verbal Learning and Verbal Behavior, 11, 671-684, 1972.

ELLIS, Nick. Vocabulary acquisition: Psychological perspectives. In: Vocabulary Acquisition Research Group. Swansea: University of Wales, 1995.

GRABE, W. \& STOLLER, F. L. Reading and vocabulary development in a second language. In Coady, J. \& Huckin, T. (Eds.), Second Language Vocabulary Acquisition. Cambridge: Cambridge University Press, 1997.

HALL, S. Using split information tasks to learn mathematics vocabulary. In Guidelines, 24 (2), 72-77, 1992.

HAYNES, m. \& BAKER, I. American and Chinese readers learning from lexical familiarization in English texts. In Huckin, T., Haynes, M. \& Coady, J. (Eds.) Second Language Reading and Vocabulary Learning. Norwood, N. J.: Ablex, 1993.

HULSTIJN, Jan H. Retention of inferred and given word meanings: Experiments in incidental learning. In Arnaud, P. J. L. \& Béjoint, H. (Eds.) Vocabulary and Applied Linguistics. Basingstoke: Macmillan, 1992.

KRASHEN, Stephen D. The Input Hypothesis: Issues and Implications. Torrance, CA: Laredo Publishing Co.,1985.

KRASHEN, Stephen D. We acquire vocabulary and spelling by reading: Additional evidence for the input hypothesis. In Modern Language Journal, 73, 440-464, 1989.

LAUFER, Batia. The lexical plight in second language reading: Words you don't know, words you think you know, and words you can't guess. In Coady, J. \& Huckin, T. (Eds.) Second Language Vocabulary Acquisition. Cambridge: Cambridge University Press, 1997.

LUPPESCU, Stuart, DAY, Richard R. Reading, dictionaries and vocabulary learning. In: HARLEY, Birgit. Lexical Issues in Language Learning. Ann Arbor: Research Club in Language Learning, 229-251, 1995.

MEARA, Paul. Vocabulary acquisition: A neglected aspect of language learning . In Language Teaching and Linguistics: Abstracts, 15, 221-246, 1980.

NAGY, W. E. \& ANDERSON, R. C. How many words are there in printed school English. In Reading Research Quaterly, 19 (3), $304-$ 330,1984

NAGY, W. E. \& HERMAN, P. A. Incidental vs. instructional approaches to increasing reading vocabulary. In Educational Perspectives, 23, 16-21, 1985.

NAGY, W. E., HERMAN, P. A. \& ANDERSON, R. C. Learning words from context. In Reading Research Quaterly, 20 (2), 233-253, 1985. 
NAGY, W. E., ANDERSON, R. C. \& HERMAN, P. A. Learning word meanings from context during normal reading. In American Educational Research Journal, 24 (2), 237-270, 1987.

NAGY, W. E. \& HERMAN, P. A. Breadth and depth of vocabulary knowledge: Implications for acquisition and instruction. In McKeown, M. G. \& Curtis, M. E. (Eds.) The Nature of Vocabulary Acquisition. Hillsdale: Lawrence Erlbaum, 1987.

NAGY, W. E. Teaching Vocabulary to Improve Reading Comprehension. Urbana: ERIC Clearinghouse on Reading \& Communication Skills, 1988.

NATION, Paul, COADY, James. Vocabulary and reading. In: CARTER, Ronald, MCCARTHY, Michael. Vocabulary and Language Teaching. New York: Longman, 97-110, 1988.

NATION, Paul, NEWTON, Jonathan. Teaching vocabulary. In: COADY, James, HUCKIN, Thomas. Second Language Vocabulary Acquisition. Cambridge: Cambridge University Press, 238-254, 1997.

PARIBAKHT, T. Sima, WESCHE, Marjorie. Vocabulary enhancement activities and reading for meaning in second language vocabulary acquisition. In: COADY, James, HUCKIN, Thomas. Second Language Vocabulary Acquisition. Cambridge: Cambridge University Press, 174-200, 1997.

PARRY, Kate. Too many words: Learning the vocabulary of an academic subject. In Huckin, T., Haynes, M. \& Coady, J. (Eds.) Second Language Reading and Vocabulary Learning. Norwood, N. J.: Ablex, 1993. (trabalho apresentado na $22^{\text {nd }}$ Annual Conference of TESOL Convention, Chicago, 1988)

SARAGI, T., NATION, I. S. P. \& Meister, G. F. Vocabulary learning and reading. In System 6 (2), 70-78, 1978.

SCHMIDT, Richard W. The role of consciousness in second language learning. In Applied Linguistics 11 (2), 129-158, 1990.

STANOVICH, K. E. \& CUNNINGHAM, A. E. Studying the consequences of literacy within a literate society: The cognitive correlates of print exposure. In Memory and Cognition 20, 51-68, 1992.

STERNBERG, R. J. Most vocabulary is learned from context. In McKeown, M. G. \& Curtis, M. E. (Eds.) The Nature of Vocabulary Acquisition. Hillsdale, NJ: Lawrence Erlbaum, 1987.

SUMMERS, Della. The role of dictionaries in language learning. In: CARTER, Ronald, MCCARTHY, Michael. Vocabulary and Language Teaching. New York: Longman, 111-125, 1988. 